\title{
Study on Integration Management and Control System of Port Cranes
}

\author{
Ping Deng, Hanbin Xiao, Ying Zhu, Shiqing Lu \\ School of Logistics Engineering, Wuhan University of Technology, Wuhan, China \\ Email: whutdp@163.com
}

Received March 5, 2012; revised April 6, 2012; accepted April 14, 2012

\begin{abstract}
This paper proposes the design scheme of Integration Management and Control System (IMCS) using the integration and optimization design theory of port cranes' management and control system based on Enterprise Resource Planning (ERP), Manufacturing Execution System (MES) and Process Control System (PCS). The functions, interface and software design of the three sub-modules of IMCS are presented. Outcomes of this research are being employed to practical port cranes' operation and management, which eliminates the island phenomenon of cranes' storage and application conditions between the management and control department. Hence, IMCS makes the integrated automatic port cranes system come true and it significantly improves the core competitiveness of the port.
\end{abstract}

Keywords: Port Cranes; IMCS; ERP; MES; PCS; Island Phenomenon

\section{Introduction}

With the standardization and modernization of logistics, ports play an increasingly important role in the international logistics service. In this situation, ports' operation efficiency is greatly challenged, which emphasizes the importance to manage and control the port equipment efficiently. Port equipment mainly refers to port cranes, which serve ships with loading and unloading containers or other types of cargoes. The management and control level of port cranes is the important embodiment of the comprehensive strength of a port. With the development of computer technology and automatic control technology, the integrated management of port equipments received fully support from technology. Port cranes' IMCS refers to using the advanced technology of computer, automatic control, network communication, database and logistics to integrate the crane operation process, storage yard management process and logistic park service with the methods of data analysis, data mining and information integration to integrate the resources of capital flow, information flow and logistics, and realizes the unified management and control of the resources. The successful application of IMCS will improve the integrated automation level of the entire port system and provide the basis for management review and decision [1].

\section{Basic Theories of Port Crane IMCS}

With the application of IMCS in various enterprises and

"Supported by "the Fundamental Research Funds for the Central Universities". the crane's development trend of automatic, informational and intelligent, this paper employs ERP, MES, PCS and other enterprise management technology into the management and control of port crane to better realize the management control and timely decision of ports. The mode of port crane management and control are transformed to make them not independent any more through combining the management decisions and site information control.

\subsection{The Combination of ERP, MES, PCS}

ERP is the abbreviations of Enterprise Resource Planning; ERP employs information technology to realize the integration of the enterprise resource management on the basis of enterprise's advanced management ideas. ERP is an enterprise management information system, which can provide trans-regional and cross-functional real-time information.

MES is the abbreviations of Manufacturing Execution System. Through information transmission, MES can optimize management of the whole production process, MES can also give some operation instruction on reduction of the cost of production process. The application of MES which can quickly respond to the change of the production process plays an important role in effectively reducing invalid production behavior in enterprise interior, and realizes the integrated cost control of production and management.

PCS is the abbreviations of Process Control System. It is mainly to realize the automatic control of crane equip- 
ment, monitor the production process, and adopt the advanced control technology to optimize setting process control system to achieve the optimal control of production process.

From Figure 1, we can conclude that the essential role of MES and the support system of network and database fully integrate the port crane ERP subsystem and PCS subsystem, and realize the unified management of business decisions and production control process, then finish the functions of target cost decomposition, product cost accounting, cost factor analysis, cost index control, production index optimization settings etc., give the operation instruction of port crane optimal control model of reducing production cost, in order to realize the port crane dynamic control and intelligent control goals.

\subsection{ERP/MES/PCS Data Transmission}

The main functions of MES include: production scheduling, resource allocation, man-hour management, document control, etc. MES connects ERP and PCS like a bridge. On the one hand, MES can provide timely and accurate data for ERP system; on the other hand, through the underlying data acquisition and analysis of PCS, MES can provide the basis and guarantee for the operation of the optimization control lines, so as to adapt to the requirement of real-time enterprises.

The process of information transmitting from ERP to MES, then to PCS is as follow: after the operation of Manufacturing Resource Planning (MRP), it is needed to process the information of the production requirements of handling operation, production resources, working calendar, operation plan inventory status etc., then ERP transfers information of production scheduling, crane

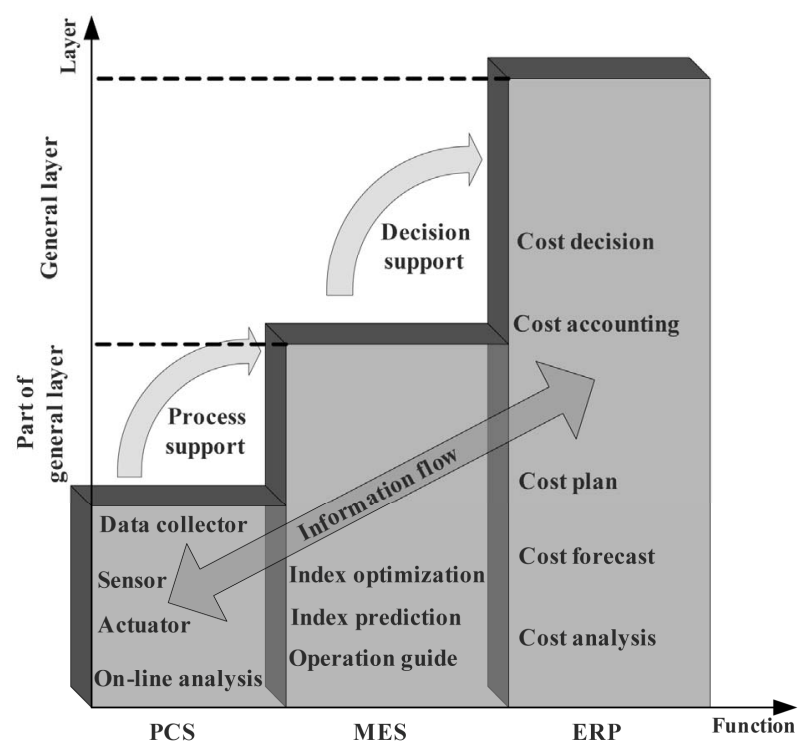

Figure 1. Three-dimensional structure of port crane IMCS based on PCS, MES and ERP. configuration, production analysis report, material shortage, production operation parameters optimization to the layer of PCS, so PCS can accordingly conduct the operation with the data obtained.

The process of information transmitting from PCS to MES, then to ERP is as follow: PCS finishes the corresponding work after accepting the work instructions of MES, then PCS will give a real-time feedback of the bottom-up information to MES, the bottom-up information includes: production progress, equipment operation parameters, workpiece clamping time, actual working hours, operation quantity, job status, task status, equipment condition, etc. Firstly, PCS process the information deeply, and then give the feedback of production status, resource state, material consumption, handling process, fault information, inventory status, and personnel distribution information to ERP system.

The transmission and feedback of information flow among the three systems guarantee the real-time information, so the crane realize the possible of the real-time planning and scheduling, dynamic intelligent management.

At the same time, the application of the integrated system of ERP, EMS and PCS changes the island phenomenon of all sorts of equipment storage application between port crane management and control; truly fulfill information system's integration and sharing.

\section{The Scheme Design of Port Crane IMCS}

\subsection{The Design Philosophy of IMCS}

The idea of the port crane IMCS design is to adopt layered implementation and system Integration methods, and it emphasizes the latter one.

1) Layered implementation. It means that industrial automation control network and integrated automation network are layered implementation on the structure and function [2]. In view of that, port cranes have realized the automation of foundation, and they also realized the industrial automation control network based on Ethernet and field bus, at the same time, the integrated automation network will consolidate each functional department within the port area, it will realize each management information system of process management, planning management, equipment operating management, human resources management, office automation, etc.

2) System integration. IMCS mainly embodies information integration and function integration. It uses the technology of computer network, workflow, real-time database, middleware, control system realizing the integration of management and control. The main contents include:

- Realizing network integration based on the original control system and management system network. 
- Getting through the data channel of the control system and management system based on the original industrial control software and relational database system.

- To establish the integrated model of management and control, studying the integrated method of management process and control process.

- To raise data integration schemes, realizing different system data's uniform access and information sharing.

- To develop production management system by applying workflow idea and middleware technology based on the data integration and the integrated model of management and control.

\subsection{The Design Objective of IMCS}

The main design goal of the port crane IMCS are to establish the distributed control system of port crane and to realize the port management information network integration, real-time monitoring for the crane operation within port area. To establish a system of highly flexible, high availability and high expansibility, realize the information sharing and transferring among crane IMCS, administrative office automation system, financial management system, warehouse management system and enterprise website. At the same time, establish the security system of software maintenance and application which includes role partition, authority distribution, user, safe and data maintenance mechanism to ensure the safe and reliable of IMCS data.

\section{The Design and Implemention of System Submodule}

\subsection{CMMS}

CMMS adopts modular design technology and realizes the operation of all lifting equipment and the omnibearing monitoring and diagnosis of their performance and working state. Hence, CMMS gives the surveillance to hoisting equipment, electric control system, driving device performance and working state. For example, CMMS can monitor AC power supply, motor control, driver operation, safety interlock, order interlock, etc. CMMS also can monitor the very heavy portal crane hoisting, the working state of cart, trolley and luffing mechanism, so the information of current, voltage, velocity and limit state can be as a imaginal and lively picture to display [3]; it conveniently provides the statistics and examining to all mechanism operating ratio, and offers the foundation for maintenance management.

The main content of CMMS are to develop different lifting equipment software, and to integrate the equipments of special industry Ethernet module on PLC controller, network switch, wireless communication device, industrial computer, server/client, UPS power, etc.

\subsection{E-MIS}

In order to let the system can provide powerful competence for information processing and data analysis, and to help managers to effectively manage port equipment, EMIS system is divided into information management module and dynamic data acquisition module through the analysis of the working process of the port. Also, E-MIS can exchange and analyze data by SQL SERVER 2000 database. The information management module includes the functions of fixed assets management, maintenance process control, the dynamic analysis of equipment fault frequency and the used fittings' frequency, safety hints, etc. which can provide all department personnel of port with strong data analysis, and can clearly understand the equipment's current state within port area. The dynamic data acquisition module can monitor the equipment working state and fault information in real-time. When a fault occurs, aided maintenance personnel will timely clear the fault, meanwhile the dynamic data acquisition module can provide statistical analysis data for information management module, such as running time, operation quantity, and current equipment state. In this way, production scheduled workers may timely understand the equipment state of whole port area, and flexible dispatch equipment according to their practical situation so as to raise the handling efficiency of port. The basic frame diagram of E-MIS is shown in Figure 2.

\subsection{CECS}

In terms of technology, lifting equipment's working characteristic is slow start, high, equipment acceleration and

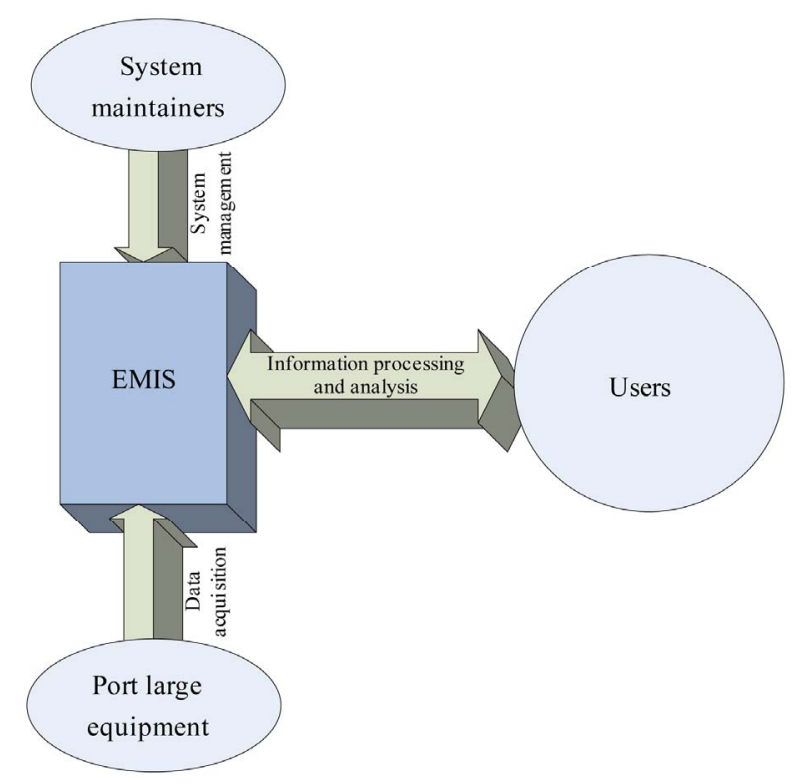

Figure 2. The basic frame diagram of E-MIS. 
deceleration smoothing, parking stationary and accurate, energy-saving operation. According to the technical requirements, port lifting equipment control system adopt many advanced control technology and driving technology in different types of crane to carry out transformation, there are some transformational ways:

- Adopt frequency conversion technology to realize the crane stable running. Employ the vector control loop method to make the whole torque of motor's inverter output when at zero-speed, the heavy weight will not downslide and hook gliding so as to realize zerospeed cross and improve lifting equipment's power factor and work efficiency. And the average energy saving rate can reach more than $20 \%$.

- Adopt intelligent electronic anti-swinging technology, combining intelligent sensor, PLC control technology and fuzzy control method of artificial intelligence to reduce the swing of the hanging goods, which can achieve precise positioning control requirements. This way can be utilized more in gantry cranes and container cranes.

- Equip the control software which has loading weight in PLC controller, the lifting velocity will automatic switching depending on the loading weight in order to achieve the operation requirements of light-load fast and heavy-load slow, which reduce the mechanical transmission impact and improve the steel structure's bearing behavior.

- System electrical control cabinet adopts plate assembly technology, which realized electrical control ca- binet standardization and scale production.

\section{Software Design of Port Crane IMCS}

\subsection{Software Design of CMMS System}

Project-developing takes several computer software development techniques, such as OPC interface technology, ADO interface technology, Access and SQL Server database programming technology, modular design technology, wired and wireless Ethernet communication technology, remote debugging and fault monitoring technology. These techniques make the large hoisting equipment collect all kinds of real-time data in operation process to local management system by Ethernet, and issue the real-time information to the remote CMMS's server of central control room by wireless network [4]. The main software uses VB to write source program code, the graphics interface adopts CorelDraw to drawing. The operating system using the popular Windows 2000 does (SP4).

CMMS system software provides the monitoring and management services according to different industry lifting equipment. There are some formed technical achievements:

- Developed six versions’ software products: quayside container crane, field bridge crane, portal crane, gantry crane, ship un-loader, stacker-reclaimer, and the six versions' software products has formed unique applied style and completed the modular design. Port crane CMMS software interface is shown in Figure 3.

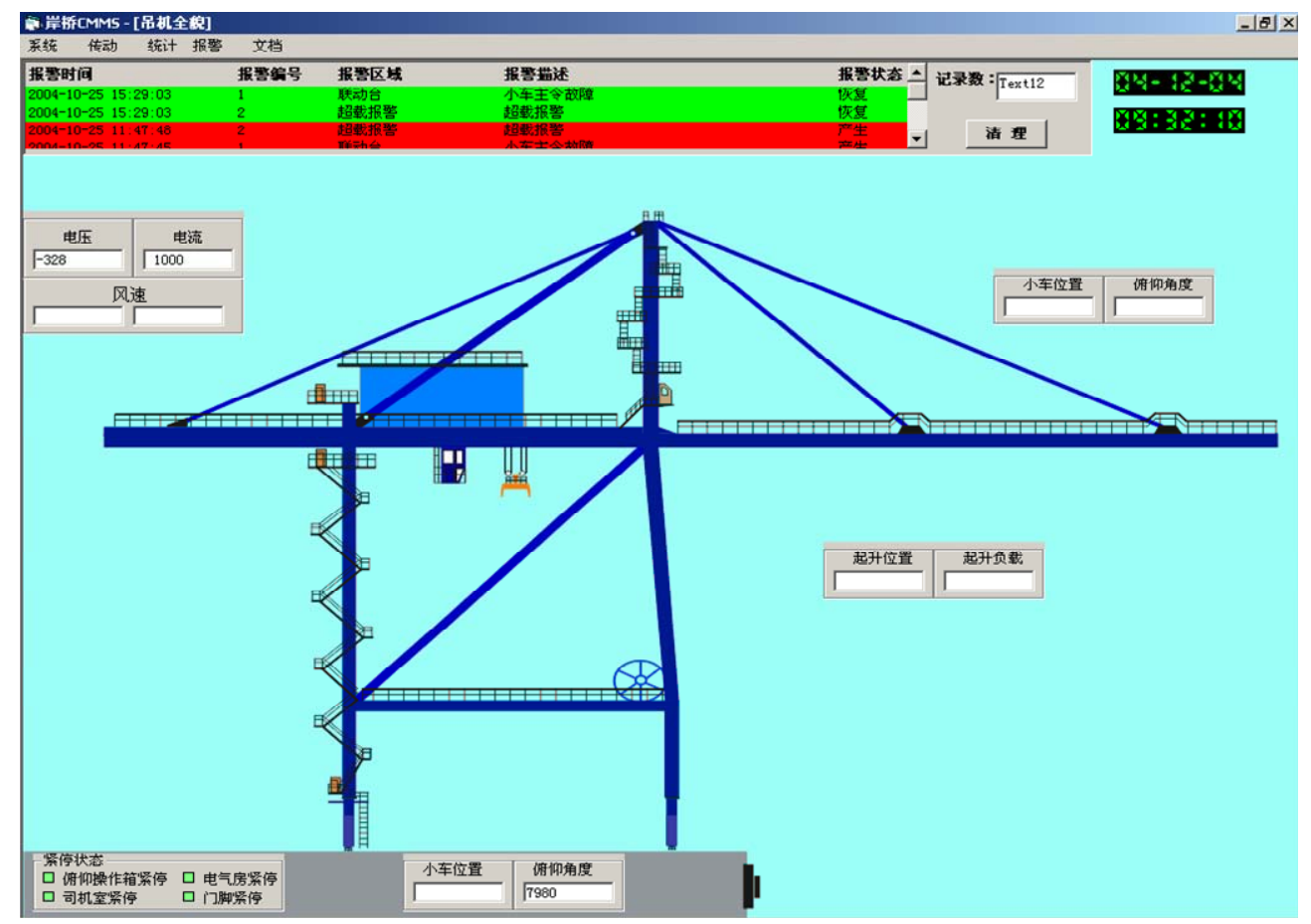

Figure 3. The version of shore container crane. 
- Verified nearly 50 projects, system operation is stable and reliable, all performance indexes meet the users' requirements and meet the demand of equipment monitoring and maintenance management, the projects have examine through the long-term use.

\subsection{Software Design of E-MIS}

E-MIS adopts Microsoft Visual Studio 2005 to develop software, database uses SQL Server 2000. The whole frame adopts C/S structure because of the demand of high real-time, which not only guarantee the system's real-time, but also increase the flexibility of operation interface.

Because the system needs to conserve the maintenance project to form a maintenance project library, so an ACCESS document can be used to conserve the maintenance project so as to share these data in several wharves, and to update data by exchanging original ACCESS document. Software structure diagram of E-MIS is shown in Figure 4.

\section{Conclusion}

The application of the port crane IMCS raises the comprehensive level of lifting equipment's site management, which can create conditions for reducing equipment downtime, maintenance cost, ensuring the safety production of maintenance target. Meanwhile it combines the equipment's running state and examination index, also it combines the equipment technical operation condition and economic operation condition, which truly fulfill comprehensive management of equipment and help the users with the overall thinking of "management efficiency". It also provides the whole reference for auxiliary leadership decision and lower operating costs. The application of the port crane IMCS has high practical value

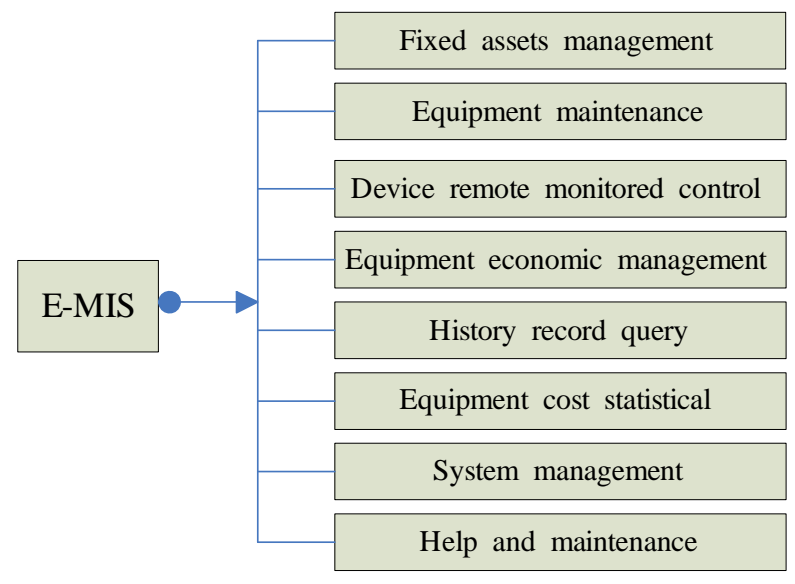

Figure 4. Software structure diagram of E-MIS.

and social value. The successful application of the port crane IMCS is a great breakthrough of mechanical equipment management and control system in port industry.

\section{REFERENCES}

[1] X. L. He, "Control and Management Integration and Integration Technology,” Shanghai Maritime University, Shanghai, 2004.

[2] T. Li, R. X. Wang, Z. F. Zhou and D. B. Wang, “The Research on Equipment Management and Control Integrative System Based on Condition Maintenance," The Machine and Hydraulic Pressure, Vol. 35, No. 11, 2007, pp. 180-182.

[3] G. H. Yang, Y. Q. Xu and H. G. Lin, “The Structure of Integrative Marine Equipment Management and Control and Its Solutions," Navigation of China, Vol. 61, No. 4, 2004, pp. 67-70.

[4] L. X. Xu and Q. Liu, "PLC Control Software System of Transport Control System in Port Handling," Port Handling, Vol. 97, No. 3, 1995, pp. 14-17. 